\title{
Diagnóstico e tratamento na avulsão dentária: uma revisão de literatura
}

\author{
Diagnosis and treatment in dental avulsion: a literature review
}

\author{
Diagnostico y tratamiento en avulsion dental: una revisión de la literatura
}

\begin{abstract}
José Milton de Aquino e Silva Neto ${ }^{1 *}$, Larissa Bezerra de Souza', Ariana Cristina Maiorano Freire', Clayton Clenisson de Carvalho Silva', Michelle Leão Bittencourt Brandão Medeiros', Tayguara Cerqueira Cavalcanti'.
\end{abstract}

\section{RESUMO}

Objetivo: Apresentar diagnósticos e tratamentos a serem seguidos para os recursos terapêuticos nos dentes avulsionados. Métodos: Tratou-se de uma revisão de literatura, possuindo abordagem descritiva com caráter informativo, onde o processo de formulação se deu através de buscas por literaturas cientificas, nas bases de dados PubMed/MedLine, LiLacs e SciELo, no período de 2015-2019, tendo como descritores booleanos: "deslocamento dentário", "processos alveolares" e "reimplantes de dentes avulsionados" nas respectivas fontes de pesquisa. Resultados: A exarticulação do dente pode provocar uma avulsão dentária, por ser uma situação anormal devido possíveis danos traumáticos ocorridos, onde se identifica pela intercorrência da deslocação do elemento dental e do processo alveolar, ocasionando problemas relevantes tanto na estrutura quanto na polpa dental. A prolongação extrabucal do dente avulsionado por grande tempo ou em formas inadequadas de conservação pode ocasionar consequências irreparáveis. Considerações Finais: A avulsão dentária é um dos problemas que pode ocasionar lesões de inserções, esse fator se estabelece em consequência do rompimento das fibras do periodonto de proteção, onde, por sua vez, ocasiona eventuais processos inflamatórios que intensificam numa larga superfície da raiz ocasionando incômodo ao paciente, deste modo os Cirurgiões Dentistas (CD) devem estar aptos a solucionar tais intercorrências.

Palavras-chave: Avulsão dentária, Protocolos clínicos, Traumatismos dentários, Reimplante.

\section{ABSTRACT}

Objective: Present diagnoses and treatments to be followed for therapeutic resources in avulsed teeth. Methods: This was a literature review, with a descriptive and informative approach, where the formulation process took place through searches for scientific literature, in the PubMed/ MedLine, LiLacs and SciELo databases, in the period 2015-2019. As descriptors: "tooth displacement", "alveolar processes" and "avulsed tooth replantations" to the boolean descriptors in the respective research sources. Results: The exarticulation of the tooth can cause a dental avulsion, as it is an abnormal situation due to possible traumatic damage, which is identified by the occurrence of dislocation of the dental element and the alveolar process, causing significant problems in both the structure and the dental pulp. The extraoral prolongation of the avulsed tooth for a long time or in inadequate preservation can cause irreparable consequences. Final Considerations: Tooth avulsion is a procedure that can cause insertion injuries, this factor is established as a result of the rupture of the fibers of the protection periodontium, which, in turn, causes possible inflammatory processes that intensify a large root surface causing much discomfort to the patient. Therefone, dentists (DC) should be able to resolve such complications.

Key Words: Tooth Avulsion, Clinical Protocols, Tooth injuries, Replantation.

\section{RESUMEN}

Objetivo: Presente las reglas y procedimientos a seguir para los recursos terapéuticos en dientes avulsados. Métodos: Esta fue una revisión de la literatura, con un enfoque descriptivo e informativo, donde el proceso de formulación fue a través de búsquedas de literatura científica, en las bases de datos PubMed/MedLine, LiLacs y SciELo, de 2015-2019. Como descriptores: "desplazamiento de los dientes", "procesos alveolares" y "replantaciones de dientes con avulsión" a los descriptores booleanos en las fuentes de investigación respectivas. Resultados: La exarticulación del diente puede causar una avulsión dental, ya que es una situación anormal debido a un posible daño traumático, que se identifica por el desplazamiento del elemento

${ }^{1}$ Centro Universitário CESMAC (CESMAC), Maceió-Alagoas. *E-mail: milton_neto_166@hotmail.com 
dental y el processo alveolar, causando problemas significativos tanto en la estructura como en la pulpa dental. La prolongación extraoral del diente avulsionado durante mucho tiempo o en una conservación inadecuada puede causar consecuencias irreparables. Consideraciones finales: La avulsión dental es un procedimiento que puede causar lesiones por inserción, este factor se establece como resultado de la ruptura de las fibras del periodonto de protección, lo que, a su vez, ocasiona procesos inflamatorios eventuales que intensifican una gran superfície de la raíz causando muchas molestias al paciente. Por lo tanto, los dentistas (DC) deberían poder resolver tales complicaciones.

Palabras Clave: Avulsión de diente, Protocolos clínicos, Traumatismo de los dientes, Reimplantación.

\section{INTRODUÇÃO}

Desde o princípio ao ser feito uma análise relacionada aos processos históricos, os traumas dentários são mais presentes em crianças e jovens que possuem faixa etária de 10 a 19 anos, possuindo como fatores etiológicos as quedas ao brincar, acidentes automobilísticos em geral, agressões físicas e os que são ocasionados por exercícios físicos sem a presença dos responsáveis (FATORI POPOVIC S, et al., 2016). Dos acidentes estabelecidos na cavidade oral, os traumatismos dentários, mais especificamente as avulsões dentárias apresentam-se com maior predomínio onde pode ser variado desde 0.5 a $16 \%$ (CHRISTOPHERSEN P, et al., 2005).

Nos últimos tempos pesquisas cientificas tem demostrado através de práticas clinicas, que traumas envolvendo os dentes, as porções alveolares e os tecidos moles adjacentes, atribui-se como sendo uma condição que ocasiona uma das principais causas da perda dental nos indivíduos. Tornando-se ainda mais impactante quando não tratado pelo Cirurgião Dentista (CD) nos primeiros momentos, ficando mais difícil a permanência do elemento dentário na cavidade bucal quando ocorre a demora ao procurar um profissional capacitado para a realização do tratamento e consequentemente o restabelecimento dos ligamentos comprometidos (BAGINSKA J e WILCZYNSKA-BORAWSKA M, 2013).

O processo recorrente as avulsões dentárias é são designados como sendo uma forma de trauma ocasionado no ligamento dentoalveolar, podendo atingir um ou mais elementos dentários, do qual, por um tempo, deslocaram-se acarretando o desagrupamento dos alvéolos. Na maioria das vezes quando o paciente chega ao consultório odontológico é observado a avulsão em apenas um exclusivo dente, sendo o mais corriqueiro os incisivos centrais superiores, sendo a dentição permanente acometida geralmente de 0,5 a $16 \%$ e na dentição decídua de 7 a 13\%, porém com menor intensidade (HUPP JR, et al., 2009).

Os traumas provocados no processo dentoalveolar é relacionado à várias intercorrências provocadas pelo agrupamento de modo impactante onde prejudicam significativamente não só os elementos dentários como também toda a estrutura que circunda o seu suporte; no que lhe concerne, a avulsão dentária especifica-se através do movimento dentário ocasionado pela deslocação do dente para o meio externo do seu sistema vital ocasionado devido as rupturas dos ligamentos alveolares, à vista disso, aconselha-se a urgência do atendimento pelo profissional capacitado para promover a integridade do dente lesionado. Geralmente, a técnica utilizada para a obtenção de um resultado satisfatório é o reimplante do elemento dentário que foi avulsionado, todavia diversos princípios precisam ser levados em consideração, principalmente na primeira consulta, obtendo uma boa anamnese da história da doença atual (RICHARDS D, 2018).

Alguns Cirurgiões Dentistas opõem-se ao processo de reimplante dos elementos dentais avulsionados, por considerarem que esse tratamento é provisório, em consequências relacionadas a forma da perspectiva e da evolução do processo de reabsorção radicular. Aliado a isto, outros profissionais, apesar disso, não executam o reimplante por causa da falta de capacitação técnica e pratica especifica para esse procedimento (RUIZ DE GOPEGUI FJ, 2003). Entretanto, pode ser observado através de inúmeros relatos clínicos que diversos Cirurgiões Dentistas realizam esse procedimento, porém existe inúmeros relatos de fracasso no tratamento, devido o emprego de protocolos incorretos (ROCHA JF, 2007). Estudos feitos por Westphalen VPD, et al. (1999) demostraram que o conhecimento técnico a respeito dessa temática precisava ser aprimorado cada vez mais pelo $C D$, aconselhando a elaborar um protocolo cientifico padronizado com o objetivo de conduzir a amostragem realizada, visto que grande parcela desses profissionais já se depararam nos consultórios com 
pacientes que precisavam de fins terapêuticos a respeito da avulsão do elemento dentário e não obtiveram de forma benéfica um resultado final satisfatório.

O conhecimento anatômico e dos processos da cavidade oral de todos os elementos dentários é importante e fundamental para adquirir conhecimentos técnicos e práticos e seguir o protocolo especifico correto para um bom planejamento na hora de tal reabilitação, com objetivo de beneficiar o resultado final do pronto atendimento ao indivíduo que é padecedor de sofrimentos acarretados pela avulsão dentária, tanto dos dentes anteriores quanto dos posteriores (BAGINSKA J e WILCZYNSKA-BORAWSKA M, 2013). Baseado nesses aspectos, o presente estudo tem como principal objetivo realizar uma revisão de literatura que demonstre as imprescindíveis decorrências que podem a vir a acontecer quando o Cirurgião Dentista depara-se com um elemento dental avulsionado e não toma as medidas cabíveis no ato do planejamento correto na hora do tratamento, além de ressaltar as possíveis consequências que o protocolo incorreto utilizado pode ocasionar, principalmente quando o reimplante acontece tardiamente acarretando desta forma um prognóstico insatisfatório.

\section{METODOLOGIA}

Para a realização da revisão de literatura, foram determinados e analisados artigos entre o período de julho de 2019 a dezembro de 2019, dos quais estavam dispostos nas bases de dados eletrônicos Literatura Latino Americana e do Caribe em Ciências da Saúde (LILACS), Literatura Internacional em Ciência da Saúde (MEDLINE) e Scientific Electronic Library Online (SCIELO) e livros que estavam disponíveis na integra na biblioteca central do Centro de Ensino Superior de Maceió (CESMAC).

Foram estabelecidas citações de textos que estavam respectivamente na língua portuguesa e inglesa, onde referiam-se aos protocolos clínicos do diagnóstico e tratamento na avulsão dentária, tendo como discernimento para o processo de exclusão a época das publicações, os artigos que não apresentavam-se na completude, séries de casos que não estavam aprofundados com a temática, ensaios não controlados e monografias, selecionando-se os textos científicos que mostrava-se na integração o real objetivo do trabalho, observando a importância da padronização no diagnóstico precoce da avulsão dentária feito pelos Cirurgiões Dentistas e o seu tratamento, tendo como principal resultado o prognostico satisfatório após o tratamento estabelecido no paciente, sendo selecionadas publicações científicas no período entre 2016 e 2019, com ênfase no intervalo entre 2017 e 2018, dispondo dos seguintes descritores: Deslocamento dentário, processos alveolares, reimplantes de dentes avulsionados e protocolos utilizados para avulsão dental.

\section{RESULTADOS E DISCUSSÃO}

Estudos realizados nas últimas décadas destacaram que o restabelecimento por completo da integridade dental pode ser estabelecido, porém esse fato acontece quando o elemento dental for rapidamente reimplantado, as primeiras horas são bastante importante para o resultado satisfatório. $O$ retardamento estabelecido para o reimplante ocasionará maior possibilidade de riscos quanto a complicações e infecções, todavia, seguindo-se os resultados obtidos através dos trabalhos clínicos modernos, foram permitidos a compreensão mais satisfatória e o mais adequado no que corresponde as cicatrizações e eventuais complicações. Elementos dentais que sofrem procedimentos com o reimplante, mesmo possuindo eventuais atrasos, conseguem, nos dias atuais possuírem inúmeros sucessos, no mínimo, no comprimento do estabelecimento das funções e/ou contribuírem para um progresso natural da mandíbula em pacientes que estão em fase de desenvolvimento, para que logo em seguida, logo após essa fase disponham de um recurso terapêutico permanente (AL SARI S, et al., 2018).

A avulsão dentária tendo sido acontecida, os ligamentos vitalícios do periodonto ocorrem grande probabilidade de sucesso no reimplante dental, sendo recomendado, na presença da improbabilidade do reimplante consecutivo, ou seja, logo após o traumatismo presente, é recomendado que referido dente possua uma boa forma de armazenamento em um ambiente úmido, e nunca em recipientes que possuam aspectos secos. Diversas são as formas citadas por autores para o seu armazenamento do elemento avulsionados nos meios citados, dos quais se destacam: os meios que possuem soluções salinas Balanceada de Hank's (SSBH), saliva, leite, vestíbulo bucal entre outros (MOHAMMAD Z, et al., 2018). O meio que utiliza a solução 
salina de Hank's é o meio mais preconizado, isso porque ele conserva até $70 \%$ dos fibroblastos presentes e fundamentais podendo o paciente ser tratado deste modo até 96 horas depois do ocorrido, entretanto poucas vezes ela encontra-se ao dispor no local do ocorrido (MOHAMMAD Z, et al., 2018). A segunda possibilidade seria o armazenamento em leite, por causa da sua osmolaridade e a sua concentração do seu potencial Hidrogeniônico, que possuem uma proporcionalidade biológica admissível (CORRÊA G, et al., 2018). Já o processo que estabelece o fluxo salivar a literatura ressalta que por ser uma forma de que existe inúmeras bactérias que podem contaminar rapidamente o hipotônico, sua recomendação não é tão favorável devido esses fatores, sendo capaz de ocasionar uma Inficionação pulpar, e ocasiona estabelecimento de reabsorção radicular no meio externo da inflamação (ODERSJÖ ML, et al., 2018). A água seria uma das últimas opções de armazenamento, sendo possuída como forma de armazenamento em elementos dentários avulsionados unicamente na ocasião em que as outras formas citadas estiverem inatingíveis naquele momento, dado que a água possui concentração hipotônica onde ocasiona lise celular e aumento das inflamações (RICHARDS D, 2018).

Foram estabelecidos critérios para a reabilitação dos elementos dentais avulsionados, tendo em vista a relação do nível de progresso do desenvolvimento radicular, isto é, identificar se o ápice radicular apresentase aberto ou fechado, levando em ponderações o processo celular que estabelece o ligamento do periodonto, considerando 3 aspectos antes mesmo de iniciar-se o tratamento do elemento avulsionado, sendo eles: analise se as células do ligamento periodontal encontram-se possivelmente factível, isso se o elemento dental for reimplantado nos primeiros minutos, no local do ocorrido, se as células periodontais conseguem ser viáveis, porém com um determinado comprometimento, isso se o dente foi armazenado de forma correta com um tempo médio de 1 hora após o acontecimento e por fim o terceiro grau que observa se as células não estão viáveis no ligamento periodontal, pois o elemento dental foi acondicionado em armazenamento seco por mais de uma hora (ANDREASEN JO e ANDREASEN FM, 2001).

A maioria das avulsões acontece principalmente nos elementos dentais anteriores, destacando os incisivos centrais superiores, devido sua anatomia possuir maior exposição no sentido vestibular, e geralmente em crianças com idades que variam de 7 a 12 anos (ANDREASEN JO e ANDREASEN FM, 2001). Segundo Baginska J, et al. (2013), a prevalência do traumatismo dentário ser maior em pacientes com idades iniciais ocorre devido ao fator das fibras presentes no ligamento periodontal estarem em processo de desenvolvimento, além do processo de formação radicular está na maioria dos casos inacabados, ou seja, com a rizogênese incompleta.

A avaliação do ligamento periodontal e da rizogênese do dente é fundamental e bastante relevante para ser analisado no atendimento emergencial, pois quando o ligamento periodontal que permaneceu aderido na área radicular não sofre ressecamento, as implicações ocasionadas pela avulsão frequentemente são mínimas (RICHARDS D, 2018). As estruturas do tecido conjuntivo frouxo que estão presentes no ligamento periodontal quando permanecem hidratadas, fazem com que sua vitalidade volte logo após o reimplante, através dos processos celulares altamente vascularizados que estão presentes no periósteo, tendo respostas inflamatórias reduzidas como consequência. Além do que, a localização do impacto e inflamações são bastante delimitadas, torna-se apropriado as restaurações, através da reposição do cemento logo após a inflamação inicial (FATORI POPOVIC S, et al., 2016).

Se por ventura o ressecamento for estabelecido em grande parte do ligamento periodontal, antes mesmo do tratamento, o sistema que engloba as células presentes fica difícil de ser restabelecido, fazendo com que ocorra uma resposta inflamatória exacerbada causada pelas células danificadas do ligamento periodontal, em uma região não mais localizada, mas sim em áreas difusas na dimensão do segmento radicular. Nesta conjuntura, existe uma elevada extensão radicular onde deverá ser reparada através de um tecido neoformado. Entretanto, os cementoblastos responsáveis pela produção de cementos celulares e acelulares, que se encontram nos feixes do ligamento periodontal, são movimentados vagarosamente, não podendo revestir a face radicular em tempo hábil, encontrando-se provavelmente só em determinadas partes. Isso fará com que o osso se estabeleça de modo direto em cima da raiz do elemento dentário (CHOU K, et al., 2014). Com relação aos procedimentos realizados e diagnósticos estabelecidos, foram analisadas e avaliadas 7 publicações mais recentes e expressivas para o decorrer do estudo (Quadro 1). 
Quadro 1 - Dados obtidos a partir de pesquisas em bases cientificas, distribuídas a partir das referências por autor, ano, país, objetivo do trabalho e os respectivos resultados.

\begin{tabular}{|c|c|c|c|}
\hline Autor (Ano) & País & Objetivo & Resultados \\
\hline $\begin{array}{c}\text { CORRÊA G, et } \\
\text { al., } 2018\end{array}$ & Brasil & $\begin{array}{l}\text { Avaliar o efeito da estratégia restauradora } \\
\text { (poste de fibra versus poste e núcleo), altura } \\
\text { coronal }(0 \mathrm{~mm} \text { vs } 2 \mathrm{~mm} \text { ) e espessura (maior } \\
\text { que } 1 \mathrm{~mm} \text { vs menor que } 1 \mathrm{~mm} \text { ) na taxa de } \\
\text { sobrevivência, resistência à fratura e } \\
\text { distribuição de tensões. }\end{array}$ & $\begin{array}{l}\text { Os grupos sem estrutura coronal remanescente apresentaram taxas de } \\
\text { sobrevida inferiores aos outros grupos. A ANOVA apresentou maiores } \\
\text { valores de carga para fraturar nos grupos com espessura coronal superior } \\
\text { a } 1 \mathrm{~mm}(p=0,0043) \text {. A análise por elementos finitos mostrou melhor } \\
\text { distribuição do estresse nos grupos com estrutura coronal remanescente e } \\
\text { restaurada com fibra. }\end{array}$ \\
\hline $\begin{array}{l}\text { ODERSJÖ ML, } \\
\text { et al, } 2018\end{array}$ & Suécia & $\begin{array}{l}\text { Coletar informações prospectivas relatadas } \\
\text { pelos pais sobre traumas dentários que } \\
\text { ocorrem em crianças de } 6 \text { meses a } 4 \text { anos de } \\
\text { idade, além de coletar dados sobre } \\
\text { complicações dos registros dentários. }\end{array}$ & $\begin{array}{l}\text { A incidência de trauma em crianças de } 6 \text { meses a } 4 \text { anos de idade foi entre } \\
12 \text { e } 48 \% \text {. Foram relatados } 41 \text { traumatismos dentários traumáticos } \\
\text { (IDTs). Destes } 24 \text { ocorreram em ambientes fechados. O motivo mais } \\
\text { comum de trauma foi a queda de acidentes. Em } 24 \text { dos } 41 \text { IDTs relatados, } \\
\text { as crianças não foram atendidas por um dentista em conexão com o } \\
\text { trauma. O motivo foi que os pais pensaram que o trauma não importava. }\end{array}$ \\
\hline $\begin{array}{c}\text { RICHARDS D } \\
2018\end{array}$ & Escócia & $\begin{array}{l}\text { Analisar a taxas de prevalência de dentição } \\
\text { permanente, dentição primária em pacientes } \\
\text { com } 12 \text { anos de idade, taxa de incidência de } \\
\text { qualquer dente para qualquer idade, razão de } \\
\text { prevalência (RP) de homem para mulher em } 12 \\
\text { anos, com intervalos de confiança de } 95 \% \text {. }\end{array}$ & $\begin{array}{l}\text { Este estudo mostrou que mais de um bilhão de pessoas vivas tiveram } \\
\text { problemas relacionados com traumatismos dentários. A avulsão dentária é } \\
\text { uma condição negligenciada que pode ser classificada em quinto lugar se } \\
\text { for incluída na lista das doenças e lesões agudas/crônicas mais frequentes } \\
\text { do mundo. }\end{array}$ \\
\hline $\begin{array}{l}\text { SOUZA XCPE, } \\
\text { et al., } 2018\end{array}$ & Brasil & $\begin{array}{l}\text { Este estudo objetivou-se em investigar fatores } \\
\text { de risco para lesões dentárias em indivíduos de } \\
\text { um serviço de referência clínica odontológica } \\
\text { para pacientes com necessidades especiais em } \\
\text { Belo Horizonte, MG, Brasil. }\end{array}$ & $\begin{array}{l}\text { A incidência de novos eventos foi de } 11,88 \% \text {. A covariável associada a um } \\
\text { risco aumentado de traumatismo dentário era uma história de lesões } \\
\text { dentárias na primeira consulta odontológica. O aumento do risco de } \\
\text { traumatismo dentário foi de } 3,59 \% \text {. Uma história de lesão dentária } \\
\text { traumática foi o fator de risco para o trauma dentário encontrado neste } \\
\text { grupo de indivíduos com deficiência no desenvolvimento. }\end{array}$ \\
\hline
\end{tabular}

REAS/EJCH | Vol.Sup.n.40 | e2657 | DOI: https://doi.org/10.25248/reas.e2657.2020 Página 5 de 9 


\begin{tabular}{|c|c|c|c|}
\hline $\begin{array}{c}\text { ODE W, et al., } \\
2018\end{array}$ & Cingapura & $\begin{array}{l}\text { O objetivo deste estudo foi examinar o impacto } \\
\text { das lesões dentárias traumáticas (IDT) nos } \\
\text { pacientes e comparar as percepções dos } \\
\text { pacientes e dentistas sobre o evento. }\end{array}$ & $\begin{array}{l}\text { A análise quantitativa mostrou que } 28 \%-55 \% \text { dos pacientes com lesões } \\
\text { dentárias traumáticas apresentaram desconforto "ocasional" a "muitas } \\
\text { vezes" durante a refeição, aumento da autoconsciência e } \\
\text { constrangimento. A análise qualitativa mostrou que os pacientes estavam } \\
\text { preocupados com a incapacidade estética, o custo do tratamento e a perda } \\
\text { potencial de dentes, mas superaram suas perspectivas negativas e } \\
\text { aceitaram as medidas de proteção prescritas. }\end{array}$ \\
\hline $\begin{array}{l}\text { AL SARI S, et } \\
\text { al., } 2018\end{array}$ & $\begin{array}{c}\text { Emirado } \\
\text { Árabes Unidos }\end{array}$ & $\begin{array}{l}\text { Avaliar o conhecimento de enfermeiros e } \\
\text { professores de educação física sobre o } \\
\text { gerenciamento emergencial de lesões } \\
\text { dentárias traumáticas (IDTs) nas escolas e } \\
\text { medir o impacto da educação em seus } \\
\text { conhecimentos. }\end{array}$ & $\begin{array}{l}\text { Melhoria significativa na escolha de procurar imediatamente atenção após } \\
\text { a avulsão dentária foi observada }(p=0,001) \text {. A deficiência inicial de } \\
\text { conhecimento sobre o gerenciamento emergencial de IDTs entre } \\
\text { professores de SN e PE foi melhorada e sustentada. }\end{array}$ \\
\hline $\begin{array}{l}\text { MOHAMMAD Z, } \\
\text { et al., } 2018\end{array}$ & Índia & $\begin{array}{l}\text { Sugerir uma nova técnica para tratar uma } \\
\text { fratura complicada da raiz da coroa. } O \\
\text { tratamento deste caso incluiu procedimento } \\
\text { endodôntico e extrusão ortodôntica para mover } \\
\text { a linha de fratura acima do nível supragengival. }\end{array}$ & $\begin{array}{l}\text { Dentre todas as lesões dentárias, as fraturas complicadas da raiz da coroa } \\
\text { (Classe VI de Andreasen) dos dentes anteriores permanentes superiores } \\
\text { são relativamente comuns. Esses dentes fraturados são frequentemente } \\
\text { considerados sem esperança e são extraídos. No entanto, se o dente } \\
\text { precisar ser retido, várias estratégias de tratamento foram propostas como } \\
\text { Um aparelho removível personalizado de Hawley com uma mola } \\
\text { modificada e um suporte Begg de ancoragem colocando-se na coroa } \\
\text { residual do dente. }\end{array}$ \\
\hline
\end{tabular}

Fonte: Neto JMAS, et al., 2019.

REAS/EJCH | Vol.Sup.n.40 | e2657 | DOI: https://doi.org/10.25248/reas.e2657.2020 Página 6 de 9 
No decorrer da fase de reconstrução do metabolismo ósseo estabelecido pelo processo fisiológico, todo o conjunto que compõem a raiz dentária ficará modificada por formação ossificada, sendo intitulado como substituição ossificante ou remodelação por substituição, podendo a avulsão dentária acarretar uma necrose da polpa dentinária, tornando o tecido afetado sujeito a propagações infecciosas de origem bacteriana (AL SARI S, et al., 2018). Se não acontecer a restauração da perfusão ou um procedimento endodôntico correto não tenha sido estabelecido, a parte constituinte da polpa encontrar-se-á impreterivelmente infeccionada; a junção de bactérias presentes no canal e as lesões localizadas no cemento e na parte externa dele, acarretará em uma reabsorção inflamatória. Com isso, as implicações ocasionadas por decorrência da avulsão são associadas de forma direta com à seriedade e à região do processo inflamatório estabelecido em cima da parte superficial da área radicular, que necessita estar refeita (MOHAMMAD Z, et al., 2018).

É inquestionável que as atividades traumáticas desenvolvem, em todos os tecidos envolvidos na fixação do dente ao osso, profundo danos aos tecidos, com a diminuição de cementoblastos presentes no ligamento periodontal, em amplas regiões da face da lesão fibro óssea; no ligamento periodontal, onde possuem como respectivas funções os processos de formação, nutricional, física e sensorial. Quando se tem avulsões traumáticas verifica-se atividades hemorrágicas e necróticas que, progressivamente, vão concedendo áreas ao fluido que possuem infecção extravascular onde dispõe grande acumulação de proteína, fragmento celular, o que concede em modificação relevante na permeabilidade normal dos menores vasos sanguíneos na área danificada e concentrações de infiltrado inflamatório, cruciais à restauração tecidual (CORRÊA G, et al., 2018).

No desenvolvimento das reparações teciduais, diversos fenômenos acontecem concomitantemente, possuindo como fundamental os acontecimentos do discernimento e redirecionamento celular. No decorrer dos reparos, uma faixa superficial radicular pode acontecer, e, se a forma de reabsorção ocorrer de maneira mais externa, a concavidade gerada tornar-se-á consertada pela degradação de um cemento reestabelecido. Os cementoblastos que estão próximos dos cementoblastos irá sucumbir os pré-cementoblastos se restabeleceram e introduziu-se com o objetivo de revestir a parte cementária. Dessa maneira, se for estabelecido o infiltrado, irá ser pequena com isso passará a ser despercebida tanto clinicamente quanto ao exame radiográfico (ODERSJÖ ML, et al., 2018).

Ao ser analisado as radiografias, elas apresentam como principais representações as modificações das imagens da anatomia das raízes por ossos radiculares. As alterações ocasionadas pelos processos inflamatórios demostram-se através da imagem radiográfica com características radiolúcidas assimétricas em todo o canal radicular. Diversas circunstâncias clínicas que conseguem sobrevir desinente de um traumatismo dental são as modificações cromáticas ou colestase neonatal da coroa, que conseguem tornar-se escurecidas. A existência de infra-oclusão ocasionada pela ausência total ou não dos ligamentos periodontais e se caracteriza devido a fusão anatômica do cemento e/ou dentina com o osso alveolar e a presença de fistulas podem também ser presentes nesses achados clínicos (ODE W, et al., 2018).

Diferentes motivos colaboram para o processo que ocorra a reabsorção da raiz do elemento dental afetado, esse acontecimento se dar devido a inúmeras bactérias presentes no ligamento do periodonto, na dentina, canais radiculares ou na polpa, do mesmo modo que as particularidades do procedimento endodôntico, quando efetuado. A proliferação bacteriana colabora diretamente com as reações inflamatórias, assim sendo, para a indução do dente a formas reabsorvidas, chegando aos túbulos dentinários poderá acarretar dois principais processos de reparo, o primeiro irá contribuir com a reabsorção da raiz se tiver processos necróticos presentes ocasionados por conta da infecção, já o segundo caso se o canal radicular possuir na região afetada tecidos normais ou inflamados, ocasionará renovação da cavidade afetada através da presença de novos cementos (RICHARDS D, 2018).

No entanto as necroses geralmente ocorrem, com restrição em rizogênese incompleta, em que a conservação papilar poderá beneficiar conservação do vigor pulpar. Quando os elementos dentais apresentam o ápice semiaberto ou aberto, a reparação do periodonto possibilita maior descomplicação no momento em que se compara aos dentes com o ápice fechado; quanto mais amplo ficar o forame apical, mais adequado encontrar-se-á o resultado do procedimento, visto que a revascularização da região pulpar é

REAS/EJCH | Vol.Sup.n.40 | e2657| DOI: https://doi.org/10.25248/reas.e2657.2020 Página 7 de 9 
viabilizada. No entanto, a infecção transmitida por bactérias gram negativas presentes no ligamento periodontal e da área pulpar descressem a possibilidade de ocorrer a revascularização. Deste modo, a conjuntura mais desagradável acarreta-se em crianças e adolescentes que possuem elementos dentais com rizogênese concluída, dado que a revascularização pulpar se realiza dificilmente e é estabelecida uma ancilose onde é acompanhada ligeiramente de reabsorção por substituição ocasionando estruturas mineralizadas (SOUZA XCPE, et al., 2018).

Depois de ser feito o processo de reimplante do elemento dental, é de suma importância que o Cirurgião Dentista prescreva a utilização de antibioticoterapia por um tempo que irá variar de 7 a 10 dias, o emprego também de antiinflamatório por 3 a 5 dias, além disto o uso de profilaxia antitetânica (AL SARI S, et al., 2018). A técnica de fixação dos dentes com auxílio de resina odontológica conhecido como esplintagem rígida é apropriada no momento que possuir ruptura dos processos alveolares simultâneo ao traumatismo referente a avulsão dentária, porém o prolongamento dessa técnica só irá ser estendida entre 4 a 8 semanas dependendo do caso particularizado de cada paciente (MOHAMMAD Z, et al., 2018). Além de todo cuidado medicamentoso pós o tratamento feito é de suma importância o cuidado com a alimentação principalmente nas primeiras duas semanas e a atenção com a higiene oral, tendo que ser feita com escovas macias de forma suave e a utilização de clorexidina a 0,12\% para a realização de bochechos nos primeiros 7 dias (RICHARDS D, 2018).

A utilização de toda essa técnica deve ser acompanhada clinicamente e radiograficamente pelo Cirurgião Dentista responsável, tendo o paciente o acompanhamento médio de 5 anos, estabelecido a princípio semanalmente até os dois meses consecutivos, em seguida o acompanhamento passa a ser semestral, e, após os dois primeiros semestres, o supervisionamento necessitará ser anualmente (MOHAMMAD Z, et al., 2018). Essa supervisão deve ser estabelecida levando-se em consideração cada etapa, visto que em alguns casos pode ocorrer o processo de reabsorção radicular, sendo está uma das consequências mais agravante relacionada a avulsão dentária. Identificando-se em três tipos distintos: Reabsorção Superficial em que a superfície do processo radicular demostra-se com uma lacuna de reabsorção recomposta por novos cementos, Reabsorção Inflamatória onde pode ser visto áreas com aspectos mais radiolúcidos em lacunas assimétrico, observando que, nesta situação especifica não existe sintomatologia clínica e Reabsorção por Substituição onde ocorre o processo de formação de anquilose devido a raiz sofre reabsorção e ser modificada por osso(AL SARI S, et al., 2018).

\section{CONSIDERAÇÕES FINAIS}

De acordo com a resolução da literatura correlata, foi possível concluir que os diagnósticos e tratamentos de avulsão dental provoca o completo deslocamento do elemento dental de seu respectivo alvéolo, estando considerada como sendo um dano bastante complexo, dos quais, é essencial que determinadas metodologias sejam estabelecidas visando as maiores chances de êxito no reimplante. Contudo, é de suma importância o estabelecimento de novos estudos clínicos mais aprofundados e detalhados para que haja melhor conhecimento sobre a temática e a medida mais aconselhável a ser tomada em casos de avulsão dental.

\section{REFERÊNCIAS}

1. AL SARI S, et al. An Educational Initiative for Dubai School Nurses and Physical Education Teachers on the Management of Traumatic Dental Injuries. J Sch Nurs 2018; 1:10.

2. ANDREASEN JO, ANDREASEN FM. Texto e atlas colorido de traumatismo dental. 3르 ed. Porto Alegre: Artmed; 2001.

3. BAGINSKA J, et al. Continuing dental education in the treatment of dental avulsion: Polish dentist's knowledge of the current IADT guidelines. Eur J Dent Educ 2013; 17: e88-92.

4. BAGINSKA J, WILCZYNSKA-BORAWSKA M. Continuing dental education in the treatment ofdental avulsion: Polish dentists' knowledge of the current IADT guidelines. Eur J Dent Educ. 2013; 17: p.88-92.

5. CHOU K, et al. Effectiveness of different in tracanal irrigation techniques in removing intracanal paste medicaments. Aust Endod J. 2014; 40(1):21-5.

6. CHRISTOPHERSEN P, et al. Avulsion of primary teeth and sequelae on permanent successors. Dent Traumatology. 2005 Dec; 21(6): 320-3.

7. CORREA G, et al. Influence of remaining coronal thickness and height on biomechanical behavior of endodontically treated teeth: survival rates, load to fracture and finite element analysis. J Appl Oral Sci 2018; 26: 55-62.

REAS/EJCH | Vol.Sup.n.40 | e2657| DOI: https://doi.org/10.25248/reas.e2657.2020 Página 8 de 9 
8. FATORI POPOVIC S, et al. Pregnancy and breast feeding: antibiotics, irrigation and pastes. Swiss Dent J 2016; 126(5):490-1.

9. HUPP JR, et al. Cirurgia Oral e Maxilofacial Contemporânea. 5.ed. Rio de Janeiro: Elsevier, 2009.

10. Mohammad Z, et al. A novel approach to extrude subgingivally fractured tooth using customized removable appliance. Int J Clin Pediatr Dent 2018; 11(1):53-7.

11. ODE W, et al. Understanding patients' and dentists' perspectives in dental trauma management: a mixed methods study. Dent Traumatol 2018; 34(4):1-6.

12. ODERSJÖ ML, et al. Incidence of dental traumatic injuries in children 0-4 years of age: a prospective study based on parental reporting. Eur Arch Paediatr Dent 2018; 19(2):107-11.

13. RICHARDS D. One billion people have experienced a traumatic dental injury. Evid Based Dent 2018; $19(2): 34-5$.

14. ROCHA JF. Nível de conhecimento do cirurgião-dentista atuante na cidade de João Pessoa acerca da avulsão dentária. [Monografia]. João Pessoa: Universidade Federal da Paraíba; 2007.

15. RUIZ DE GOPEGUI FJ. Incisivo central permanente avulsionado. Conducta y caso clínico. RCOE. 2003; 8(4): 429437.

16. SOUZA XCPE, et al. What Increases the risk of dental traumatism in patients with developmental disabilities? Braz Dent J. 2018; 29(2):154-8.

17. WESTPHALEN VPD, et al. Avulsão dentária: condutas clínicas. JBC- J Bras Clin Estét Odont 1999;3(15):79-83. 